\title{
Symptom burden \& quality of life among patients receiving second-line treatment of metastatic colorectal cancer
}

Mark S Walker ${ }^{1,4^{*}}$, Elaine Yu Pharm², Jiandong Kerr ${ }^{1}$, Yeun Mi Yim² ${ }^{2}$ Edward J Stepanski ${ }^{1}$ and Lee S Schwartzberg ${ }^{3}$

\begin{abstract}
Background: Bevacizumab (B) and cetuximab (C) are both approved for use in the treatment of metastatic colorectal cancer $(\mathrm{mCRC})$ in the second-line. We examined patient reported symptom burden during second-line treatment of $\mathrm{mCRC}$.

Methods: Adult $m C R C$ patients treated in the second-line setting with a regimen that included $B, C$, or chemotherapy only $(\mathrm{O})$ and who had completed $\geq 1$ Patient Care Monitor (PCM) surveys as part of routine clinical care were drawn from the ACORN Data Warehouse. Primary endpoints were rash, dry skin, itching, nail changes, nausea, vomiting, fatigue, burning in hands/feet, and diarrhea. Linear mixed models examined change in PCM scores across $B, C$ and $O$ ( $B=$ reference).

Results: 182 patients were enrolled (B: $n=106, C: n=38, O: n=38$ ). Patients were $51 \%$ female, $67 \%$ Caucasian, with mean age of 62.0 ( $S D=12.6)$. Groups did not differ on demographic or clinical characteristics. The most common second-line regimens were FOLFIRI \pm B or C (23.1\%) and FOLFOX \pm B or C (22.5\%). Results showed baseline scores to be strongly predictive of second-line symptoms across all PCM items (all p's $<.0001$ except for Rash, $p=.0013$ ). Controlling for baseline, patients on B tended to have more stable and less severe symptoms. Patients on $C$ had more severe rash, dry skin, and itching and had nail change scores that worsened faster than did B patients.
\end{abstract}

Conclusions: Patients receiving second-line treatment for mCRC with B report less symptom burden, especially dermatologic, compared to patients treated with C.

Keywords: Bevacizumab, Cetuximab, Chemotherapy, Health outcomes, Dermatologic symptoms

\section{Background}

The American Cancer Society estimates that approximately 141,210 people will be diagnosed with colorectal cancer (CRC) in the United States in 2011 with roughly 49,380 people dying of the disease during the same time frame [1]. CRC is the third most commonly diagnosed cancer among both men and women and the third leading cause of cancer death overall. Incidence and death rates for CRC increase with age with $90 \%$ of new cases and $94 \%$ of deaths occurring in individuals 50 years of age and older [1].

\footnotetext{
* Correspondence: mwalker@acorncro.com

${ }^{1}$ ACORN Research, LLC, Memphis, TN, USA

${ }^{4} 6555$ Quince, Suite 400, Memphis, TN 38119, USA

Full list of author information is available at the end of the article
}

CRC is a cancer that starts in the large intestine or the rectum. Cancer cells eventually spread to nearby lymph nodes and subsequently to more remote lymph nodes and other organs in the body with the liver and lungs being the most common metastatic sites. Approximately $30 \%$ of all patients with CRC have metastatic disease at diagnosis, and between $40 \%$ and $65 \%$ of all patients diagnosed with CRC will eventually develop metastatic or advanced disease $[2,3]$.

The management of patients with metastatic colorectal cancer (mCRC) has changed dramatically over the last decade. Historically, 5-fluorouracil (5-FU) was the only active agent in CRC. The introduction of several new chemotherapeutic (irinotecan, oxaliplatin) and biologic agents (cetuximab, bevacizumab, panitumumab) into

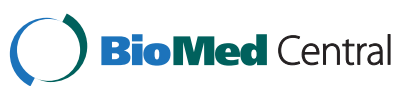

(c) 2012 Walker et al.; licensee BioMed Central Ltd. This is an Open Access article distributed under the terms of the Creative Commons Attribution License (http://creativecommons.org/licenses/by/2.0), which permits unrestricted use, distribution, and reproduction in any medium, provided the original work is properly cited. 
clinical practice have resulted in significant gains in response rates and overall survival [4-6].

The therapies recommended by the National Comprehensive Cancer Network (NCCN) after the first progression in patients who have received prior 5-FU/ leucovorin (LV) based or capecitabine based therapies are dependent on the initial treatment regimen $[7,8]$. If FOLFOX or CapeOx based therapies are used as first-line, FOLFIRI, with or without cetuximab or panitumumab (KRAS wild type tumor only), and irinotecan in combination with cetuximab (KRAS wild type tumor only) or as a single agent is recommended. In patients who received a FOLFIRI based regimen as the first-line therapy, FOLFOX or CapeOx, cetuximab plus irinotecan, or single agent cetuximab or panitumumab (for those not appropriate for the combination with irinotecan) are recommended options. For patients who received 5-FU/LV or capecitabine without oxaliplatin or irinotecan as initial therapy, options after first progression include FOLFOX, CapeOx, FOLFIRI, single agent irinotecan, or irinotecan plus oxaliplatin. For patients who received FOLFOXIRI as initial therapy, cetuximab plus irinotecan or cetuximab or panitumumab alone are recommended options for those with wild-type KRAS gene. NCCN guidelines also note that bevacizumab, if not used in initial therapy, may be appropriate to add to chemotherapy following progression of metastatic disease.

Treatments for mCRC are mainly palliative. They seek to increase the duration, and maintain or improve the quality of the patient's remaining life, a difficult task given the toxicity of the given chemotherapy combinations [5]. The addition of bevacizumab or cetuximab to these regimens may result in somewhat different toxicity profiles. Package inserts for both products report that common reactions include headache and diarrhea $[9,10]$. Bevacizumab labeling also reports epistaxis (nosebleed), hypertension, rhinitis, proteinuria, taste alteration, dry skin, rectal hemorrhage, lacrimation disorder, back pain and exfoliative dermatitis to occur in $\geq 10 \%$ of cases [10]. Cetuximab labeling reports rash, pruritus, nail changes and infection to occur in $\geq 25 \%$ of cases [9].

Research has suggested health-related quality of life (HRQoL) is positively associated with subsequent survival duration [11-13], making it an important consideration in decisions related to treatment of patients with metastatic disease. The total number of side effects and the intensity of specific toxicities, most notably fatigue, insomnia, nausea, vomiting and diarrhea, have been shown to markedly affect patient satisfaction with HRQoL [14-17]. The specific effect of other toxicities, such as dermatologic toxicities, on patient satisfaction with HRQoL is less well understood although they are known to occur with frequency in some treatments $[18,19]$.
Although many studies have examined HRQoL among patients receiving first-line treatment of mCRC [20-22], few have examined symptom burden and HRQoL in the second-line. In the few that have, all have been conducted in the clinical trial setting. In addition, few studies have conducted multi-regimen comparisons involving recommended therapies. The goal of the current study was to describe symptom burden among patients treated for $\mathrm{mCRC}$ in community settings who received second-line regimens that contained (1) bevacizumab, (2) cetuximab or (3) chemotherapy regimens without the addition of a monoclonal antibody (designated Chemotherapy Only).

\section{Materials and methods \\ Patients \& setting}

Potentially eligible patients were identified through review of electronic records of patients at community oncology practices represented in the ACORN Data Warehouse. The ACORN Data Warehouse contains electronic medical record, billing, and patient reported outcome data from 13 community oncology practices. Data are extracted electronically, refreshed weekly, and used as source data for cancer research. Medical charts were subsequently reviewed to determine final study eligibility. The final study sample was drawn from patients in seven affiliated practices. All study procedures were approved by IntegReview, a commercial IRB in Austin, TX.

Patients were eligible if they were at least 18 years of age, had a confirmed diagnosis of stage IV colorectal cancer, had experienced at least one disease progression after diagnosis with $\mathrm{mCRC}$, and received second-line treatment with a qualifying regimen. Qualifying regimens were those that included bevacizumab, cetuximab, or chemotherapy alone without monoclonal antibodies. We considered regimens that included panitumumab, but the sample of patients who received second-line panitumumab was too small for meaningful comparison. Eligibility also required that patients had completed at least one Patient Care Monitor (PCM) assessment, described further below, during the period in which they were receiving second-line treatment for $\mathrm{mCRC}$.

\section{Primary study endpoint}

The primary study endpoint was patient reported outcomes as indicated by the PCM assessment. This included individual PCM items such as 'rash,' 'dry skin,' 'Itching,' 'nail changes,' 'nausea,' 'vomiting,' 'diarrhea, and 'burning sensation in hands or feet.' It also included PCM index scores, described below. Although other symptoms were of interest for the study, the range of items under consideration was limited by those available as part of the PCM. The focus of reporting in this paper is the individual PCM items that describe specific patient symptoms, listed above. 
PCM, version 2.0, is an 86-item self-report measure that assesses physical symptoms, psychological symptoms and physical functioning, and asks patients to rate the severity of symptoms on an 11 point (0 to 10) Likert-type scale, where higher scores reflect more severe symptoms. The PCM is administered via touch screen tablet personal computer as a routine part of care at participating community oncology practices. In addition to scores on individual PCM items, the PCM produces standardized index scores (T scores) for six screening scales in which higher scores denote more severe symptoms. The indices are: General Physical Symptoms, Treatment Side Effects, Despair and Depression, Acute Distress, Impaired Ambulation, and Impaired Performance. The PCM has been shown to be valid for assessing symptom burden and HRQoL in cancer patients and has been used in a number of studies [23-27]. The focus of this study was on symptom burden.

\section{Other measures}

Demographic characteristics included age, gender, ethnicity, body mass index, and geographic location. Clinical characteristics included performance status, primary CRC site, sites of metastases, tumor histology, KRAS status if available, and the presence vs. absence of documented evidence for KRAS testing prior to the start of second-line treatment. Treatment characteristics included first and second-line chemotherapy and/or targeted therapy regimens, including duration of first-line therapy.

\section{Statistical methods}

The underlying populations of patients treated with bevacizumab, cetuximab, or chemotherapy alone from which the sample was drawn were not of equal size; resulting samples in each treatment group were therefore expected to be unequal. In particular, we were aware that more patients with bevacizumab experience were available, and planned for a larger sample in this group to provide increased statistical power for group comparisons.

Descriptive statistics were generated for all demographic, disease, and treatment characteristics. Frequencies and percentages were generated for categorical variables and the mean, standard deviation, median, minimum, and maximum were generated for continuous variables. Chisquare tests of independence and Fisher's Exact test were used to compare groups across levels of categorical variables. $\mathrm{T}$ tests and analysis of variance were used to compare groups on continuous variables.

Linear mixed models were employed to examine change in PCM endpoints over time during the second-line period, across Bevacizumab vs. Cetuximab vs. Chemotherapy Only groups, with the Bevacizumab group defined as the reference for group comparison. Models employed restricted maximum likelihood estimation, with random intercept and random slope for regression of PCM endpoints on interval since starting second-line chemotherapy. Methods generally followed those reported by Cnaan and Little [28,29].

Models included as predictors the interval in days between the start of second-line treatment and the PCM survey (Interval), the treatment group (Group), and the interaction of these two terms. These were included in the models irrespective of statistical significance. Each model also considered the following predictors for inclusion in the model: race group, age at start of second-line therapy, duration in days of first-line therapy, body mass index, whether the patients were receiving chemotherapy or targeted therapy at the time of the PCM survey, first-line treatment regimen as containing oxaliplatin vs. irinotecan vs. neither, and second-line chemotherapy regimen as containing oxaliplatin vs. irinotecan vs. neither.

A second set of models were generated which additionally controlled for baseline PCM values. Baseline values were defined as the PCM score closest in time to the start date of second-line therapy, and within 14 days of the start of second-line therapy. Only patients with a qualifying baseline value were included in this additional analysis. All statistical tests were interpreted at alpha $=.05$, two tailed, and no adjustment was made for multiple comparisons.

\section{Results}

\section{Sample development}

A total of 696 potentially eligible patients were identified, $14.7 \%$ of whom on review did not have metastatic disease, or did not have CRC. Primary reasons for exclusion in the remaining potentially eligible subjects included no evidence of disease progression after diagnosis with mCRC (17.4\%), initiating second-line therapy prior to January 1 , 2007 (23.3\%) and insufficient PCM data (10.5\%). A total of 182 patients met all eligibility criteria, and represented the final study sample. Figure 1 shows sample development.

\section{Study population}

The sample was largely Caucasian (67.6\%) with a mean age of $62.0(\mathrm{SD}=12.6)$ years. About half of the sample was female (51.1\%). Three quarters of patients resided in the southern United States. By definition, all patients had stage IV disease. The most common primary sites of disease were sigmoid colon, cecum, and ascending colon. More than $90 \%$ of patients had adenocarcinoma. The predominant sites of metastasis were liver (65.4\%), lung (46.7\%), and the peritoneum (23.6\%). KRAS test status was unavailable in $55 \%$ of patients, primarily due to the fact that $52 \%$ of patients initiated second-line therapy prior to June 2008. Among those patients where KRAS testing was known to occur $(\mathrm{N}=44), 45.5 \%$ had mutant results. There were no significant differences in any of the demographic or clinical characteristics across the three regimen groups. 


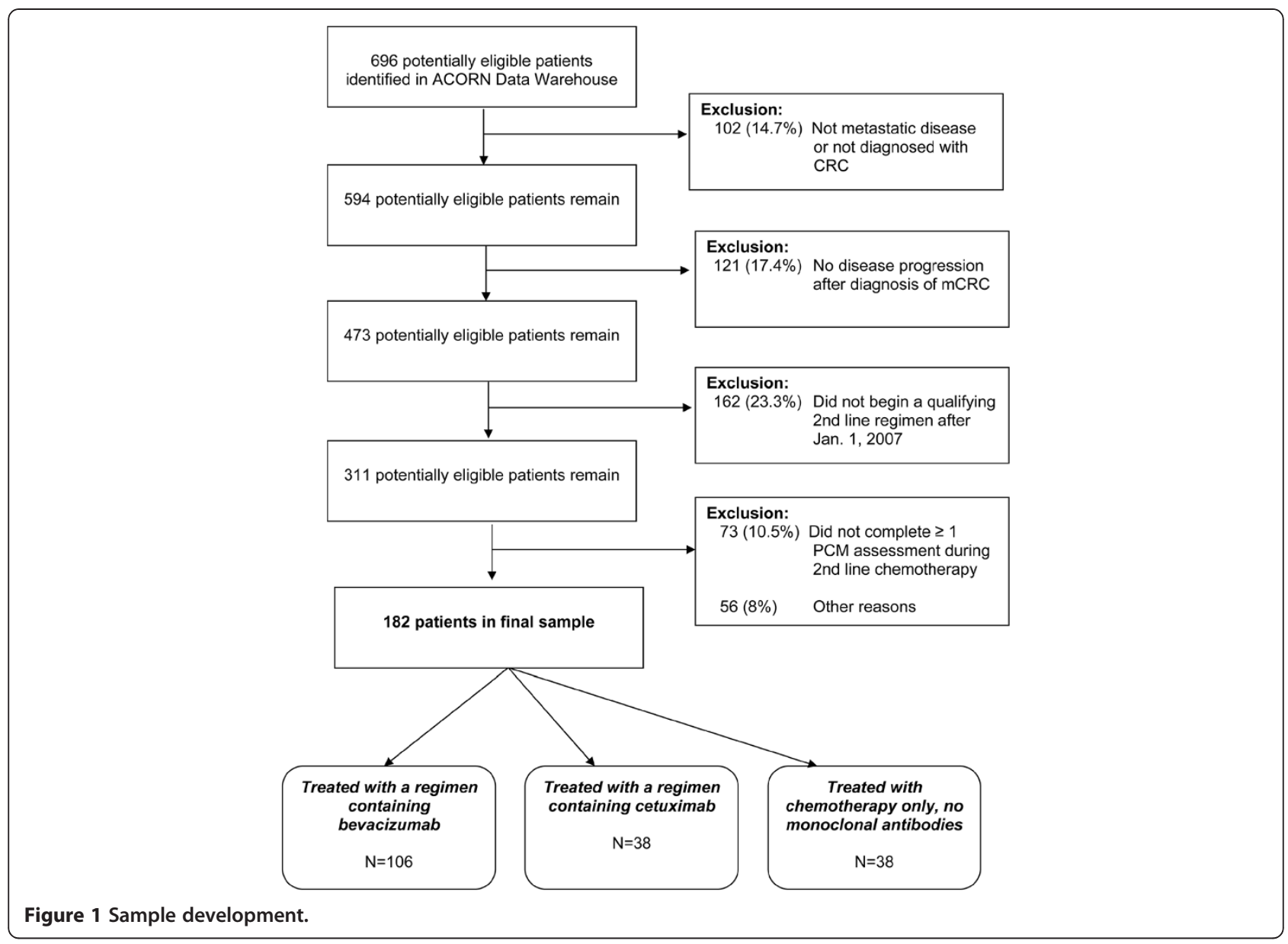

Fifty eight percent of the sample used a second-line regimen containing bevacizumab, $21 \%$ used a secondline regimen containing cetuximab, and $21 \%$ used a chemotherapy regimen that did not include a monoclonal antibody. Note, however, that the study sample was a convenience sample-patients were not randomly selected. The distribution of second-line regimens observed in this sample is therefore not assumed to reflect the population distribution of second-line regimens.

The mean duration of first-line therapy across all groups was 212.5 days ( $\mathrm{SD}=162$ days), and this did not significantly vary across second-line regimen group $(\mathrm{p}=.136)$. The mean duration of second-line therapy across all groups was 144.7 days $(\mathrm{SD}=116.4)$, but 17 patients had not finished second-line therapy at the time of data collection. Time to event analysis of the duration of second-line therapy was therefore conducted, to provide an unbiased estimate of the duration. This analysis showed the median duration of second-line therapy to be 124 days (Chemotherapy Only: 100 days, Cetuximab: 113 days, Bevacizumab: 136 days). A log rank test comparing duration across second-line treatment groups showed the group differences in duration of second-line therapy to be statistically significant $(\mathrm{p}=.0399)$.

Cetuximab patients were more likely than expected to have received an irinotecan-based regimen $\left(\chi^{2}\right.$ $(186,2)=8.765, \mathrm{p}=.0129)$. Nearly all patients who received an oxaliplatin-based regimen were in the Bevacizumab group, with about half of Bevacizumab patients receiving oxaliplatin, compared with about $11 \%$ in the combined Chemotherapy Only and Cetuximab groups $\left(\chi^{2}(186,2)=31.004, \mathrm{p}<.001\right)$. Bevacizumab patients were also much more likely to have used 5 -FU based regimens $\left(\chi^{2}(186,2)=21.548, \mathrm{p}<.001\right)$. There was no significant difference in the rate at which patients received capecitabine as part of their regimen.

Additional detail on the demographic, clinical and treatment characteristics of the population, by treatment group, are reported in Tables 1 and 2.

\section{Patient care monitor assessment}

There were 1236 valid PCM assessments available from the 182 patients in the study. Baseline scores were available for $90.6 \%$ of patients $(n=165)$, who provided a total 
of 1156 PCM assessments for analysis that controlled for baseline symptom scores.

\section{Moderate to severe symptomatology}

Many patients experienced moderate to severe symptoms, defined by PCM item scores $\geq 4$, at some point during second-line therapy. Fatigue was the most common, occurring at moderate to severe levels in $67 \%$ of patients. The Cetuximab group reported a significantly higher rate of moderate to severe dry skin $(\mathrm{p}<.0001)$, itching $(\mathrm{p}=.0028)$, and rash $(\mathrm{p}<.0001)$, with rash rates of $60.5 \%$ in the Cetuximab group vs. $7.5 \%$ in the Bevacizumab group. Patients on Chemotherapy Only had a higher rate of moderate to severe nausea $(p=.0485)$ than the Bevacizumab group, and tended to have a higher rate of physical pain $(\mathrm{p}=.0564)$ than the Bevacizumab group. The Cetuximab group tended to have a lower rate of moderate to severe problems with sweating than did the Bevacizumab group $(\mathrm{p}=.0637)$. Groups did not differ significantly on other symptoms.

\section{Linear mixed models analysis of PCM items Baseline analyses}

Linear mixed models were run twice, with one set of models controlling for baseline scores and a second set of models not controlling for baseline scores. Treatment group was significant in almost all of the models that did not control for baseline scores. However, after inclusion of baseline scores, those results changed notably as baseline symptom burden scores were the strongest predictor of

Table 1 Demographic \& clinical characteristics by treatment group

\begin{tabular}{|c|c|c|c|c|}
\hline Variables & Chemotherapy only $\mathrm{N}=38$ & Cetuximab N = 38 & BevacizumabN = 106 & Total $\mathrm{N}=182$ \\
\hline \multicolumn{5}{|l|}{ Gender, n (\%) } \\
\hline Female & $23(60.5 \%)$ & $14(36.8 \%)$ & $56(52.8 \%)$ & $93(51.1 \%)$ \\
\hline \multicolumn{5}{|l|}{ Age (years) } \\
\hline Mean (SD) & $60.8(14.7)$ & $64.6(11.2)$ & $61.5(12.3)$ & $62.0(12.6)$ \\
\hline \multicolumn{5}{|l|}{ US Region, n (\%) } \\
\hline Northeast & $4(10.5 \%)$ & $3(7.9 \%)$ & $3(2.8 \%)$ & $10(5.5 \%)$ \\
\hline Midwest & $0(0.0 \%)$ & $2(5.3 \%)$ & $4(3.8 \%)$ & $6(3.3 \%)$ \\
\hline South & $27(71.1 \%)$ & $28(73.7 \%)$ & $83(78.3 \%)$ & $138(75.8 \%)$ \\
\hline West & $7(18.4 \%)$ & $5(13.2 \%)$ & $16(15.1 \%)$ & $28(15.4 \%)$ \\
\hline \multicolumn{5}{|l|}{ Race, n (\%) } \\
\hline White & $26(68.4 \%)$ & $26(68.4 \%)$ & $71(67.0 \%)$ & $123(67.6 \%)$ \\
\hline Minority & $12(31.6 \%)$ & $12(31.6 \%)$ & $35(33.0 \%)$ & 59 (32.4\%) \\
\hline Sub-Total & $38(100 \%)$ & $38(100 \%)$ & $106(100 \%)$ & $182(100 \%)$ \\
\hline \multicolumn{5}{|l|}{ BMl } \\
\hline Mean (SD) & $26.3(6.2)$ & $28.9(5.2)$ & $26.9(5.4)$ & $27.2(5.6)$ \\
\hline \multicolumn{5}{|l|}{ Disease Stage, n (\%) } \\
\hline Stage IV & $38(100 \%)$ & $38(100 \%)$ & $106(100 \%)$ & $182(100 \%)$ \\
\hline \multicolumn{5}{|l|}{ ECOG, n (\%) } \\
\hline 0 & $8(21.1 \%)$ & $10(26.3 \%)$ & 19 (17.9\%) & $37(20.3 \%)$ \\
\hline 1 & $8(21.1 \%)$ & $6(15.8 \%)$ & $19(17.9 \%)$ & $33(18.1 \%)$ \\
\hline $2+$ & $2(5.3 \%)$ & $3(7.9 \%)$ & $3(2.8 \%)$ & $8(4.4 \%)$ \\
\hline Text indication of impairment & $4(10.5 \%)$ & $2(5.3 \%)$ & $14(13.2 \%)$ & $20(11.0 \%)$ \\
\hline No text indication of impairment & $16(42.1 \%)$ & $17(44.7 \%)$ & $51(48.1 \%)$ & $84(46.2 \%)$ \\
\hline \multicolumn{5}{|l|}{ Sites of Distant Metastasis, n (\%) * } \\
\hline Liver & $24(63.2 \%)$ & $24(63.2 \%)$ & $71(67.0 \%)$ & $119(65.4 \%)$ \\
\hline Lung & $14(36.8 \%)$ & $21(55.3 \%)$ & $50(47.2 \%)$ & $85(46.7 \%)$ \\
\hline Peritoneum & $9(23.7 \%)$ & $6(15.8 \%)$ & $28(26.4 \%)$ & $43(23.6 \%)$ \\
\hline Small intestine & $0(0 \%)$ & $0(0 \%)$ & $4(3.8 \%)$ & $4(2.2 \%)$ \\
\hline Other & $16(42.1 \%)$ & $15(39.5 \%)$ & $47(44.3 \%)$ & $78(42.9 \%)$ \\
\hline Not documented & $0(0 \%)$ & $0(0 \%)$ & $1(0.9 \%)$ & $1(0.5 \%)$ \\
\hline
\end{tabular}

\footnotetext{
* Patients may be represented on more than one row.
} 
Table 2 Treatment characteristics by second-line treatment group

\begin{tabular}{|c|c|c|c|c|}
\hline & $\begin{array}{l}\text { Chemotherapy only } \\
\qquad \mathrm{N}=\mathbf{3 8}\end{array}$ & Cetuximab N = 38 & Bevacizumab N = 106 & Total $\mathrm{N}=182$ \\
\hline \multicolumn{5}{|l|}{ Regimen used in 1st line therapy, $n(\%)$} \\
\hline Oxaliplatin based & $5(13 \%)$ & $4(11 \%)$ & $54(51 \%)$ & $63(35 \%)$ \\
\hline Irinotecan based & $21(55 \%)$ & $28(74 \%)$ & $40(38 \%)$ & $89(49 \%)$ \\
\hline Neither Oxaliplatin nor Irinotecan based & $12(32 \%)$ & $6(16 \%)$ & $12(11 \%)$ & $30(16 \%)$ \\
\hline \multicolumn{5}{|l|}{ Regimen used in 2nd line therapy, $n$ (\%) } \\
\hline $5-\mathrm{FU}$ & $0(0.0 \%)$ & $0(0.0 \%)$ & $1(0.9 \%)$ & $1(0.5 \%)$ \\
\hline 5-FU / AMG 706 / Irinotecan / Leucovorin & $1(2.6 \%)$ & $0(0.0 \%)$ & $0(0.0 \%)$ & $1(0.5 \%)$ \\
\hline 5-FU / Gamma Interferon / Irinotecan / Leucovorin & $0(0.0 \%)$ & $0(0.0 \%)$ & $1(0.9 \%)$ & $1(0.5 \%)$ \\
\hline 5-FU / Gamma Interferon / Leucovorin & $2(5.3 \%)$ & $0(0.0 \%)$ & $0(0.0 \%)$ & $2(1.1 \%)$ \\
\hline 5-FU / Interferon / Leucovorin & $2(5.3 \%)$ & $0(0.0 \%)$ & $0(0.0 \%)$ & $2(1.1 \%)$ \\
\hline 5-FU / Irinotecan / Leucovorin & $6(15.8 \%)$ & $13(34.2 \%)$ & $29(27.4 \%)$ & $48(26.4 \%)$ \\
\hline 5-FU / Irinotecan / Leucovorin / Oxaliplatin & $0(0.0 \%)$ & $0(0.0 \%)$ & $5(4.7 \%)$ & $5(2.7 \%)$ \\
\hline 5-FU / Irinotecan / Leucovorin / Oxaliplatin / Capecitabine & $0(0.0 \%)$ & $0(0.0 \%)$ & $2(1.9 \%)$ & $2(1.1 \%)$ \\
\hline 5-FU / Irinotecan / Leucovorin / Capecitabine & $2(5.3 \%)$ & $0(0.0 \%)$ & $4(3.8 \%)$ & $6(3.3 \%)$ \\
\hline 5-FU / Leucovorin & $2(5.3 \%)$ & $1(2.6 \%)$ & $3(2.8 \%)$ & $6(3.3 \%)$ \\
\hline 5-FU / Leucovorin / Oxaliplatin & $3(7.9 \%)$ & $3(7.9 \%)$ & $38(35.8 \%)$ & $44(24.2 \%)$ \\
\hline 5-FU / Leucovorin / Oxaliplatin / Capecitabine & $0(0.0 \%)$ & $0(0.0 \%)$ & $1(0.9 \%)$ & $1(0.5 \%)$ \\
\hline Bevacizumab Only & $0(0.0 \%)$ & $0(0.0 \%)$ & $2(1.9 \%)$ & $2(1.1 \%)$ \\
\hline Cetuximab Only & $0(0.0 \%)$ & $4(10.5 \%)$ & $0(0.0 \%)$ & $4(2.2 \%)$ \\
\hline Floxuridine / Irinotecan & $1(2.6 \%)$ & $0(0.0 \%)$ & $0(0.0 \%)$ & $1(0.5 \%)$ \\
\hline Irinotecan & 11 (28.9\%) & $13(34.2 \%)$ & $3(2.8 \%)$ & $27(14.8 \%)$ \\
\hline Irinotecan / Oxaliplatin / Capecitabine & $0(0.0 \%)$ & $0(0.0 \%)$ & $1(0.9 \%)$ & $1(0.5 \%)$ \\
\hline Irinotecan / Capecitabine & $0(0.0 \%)$ & $2(5.3 \%)$ & $3(2.8 \%)$ & $5(2.7 \%)$ \\
\hline Oxaliplatin / Capecitabine & $2(5.3 \%)$ & $1(2.6 \%)$ & $7(6.6 \%)$ & $10(5.5 \%)$ \\
\hline Capecitabine & $6(15.8 \%)$ & $1(2.6 \%)$ & $6(5.7 \%)$ & $13(7.1 \%)$ \\
\hline
\end{tabular}

second-line symptom burden across all measured items (all p's <.0001 except for Rash, p = .0013).

\section{Rash}

As evidenced in Figure 2, patients in the Bevacizumab group had significantly $(\mathrm{p}<.0001)$ lower (better) Rash scores than did patients in the Cetuximab group and nominally lower scores than patients in the Chemotherapy Only group. Rash scores in the Cetuximab group fell faster than those in the Bevacizumab group $(\mathrm{p}=.019)$, albeit from a higher level. It should be noted that scores in the Bevacizumab group were near enough to zero that they had little room to fall. Although baseline rash scores significantly predicted rash scores during second-line therapy $(\mathrm{p}=.0013)$, membership in the Cetuximab group was a very strong predictor. Longer duration of first-line therapy was associated with less severe rash symptoms $(\mathrm{p}=.0375)$. Patients on either irinotecan or oxaliplatin had less severe symptoms $(\mathrm{p}=.0001, \mathrm{p}=.0005$, respectively) than patients on neither agent.

\section{Dry skin}

There was a significant effect of Group $(\mathrm{p}<.0013)$, Interval $(\mathrm{p}=.01)$, and the interaction of the two $(\mathrm{p}=.0128)$ on Dry Skin scores (Figure 3), controlling for baseline. This suggests that Bevacizumab and Chemotherapy Only patients had low and stable scores whereas Cetuximab patients had Dry Skin scores that were significantly higher than the Bevacizumab group $(\mathrm{p}=.0008)$, and that also were changing (worsening) at a rate significantly different from the Bevacizumab group $(\mathrm{p}=.0037)$. Baseline scores were also a significant predictor in this model. Baseline adjusted scores for the Chemotherapy Only group were comparable to those of the Bevacizumab group whereas, when not controlled, they appeared significantly elevated. The implication of this result is that Chemotherapy Only patients may be more likely than Bevacizumab patients to enter therapy with elevated Dry Skin ratings. For those who do, elevated scores may persist. However, it is the elevated baseline scores that drive elevated scores during second-line therapy, not their group membership. In 


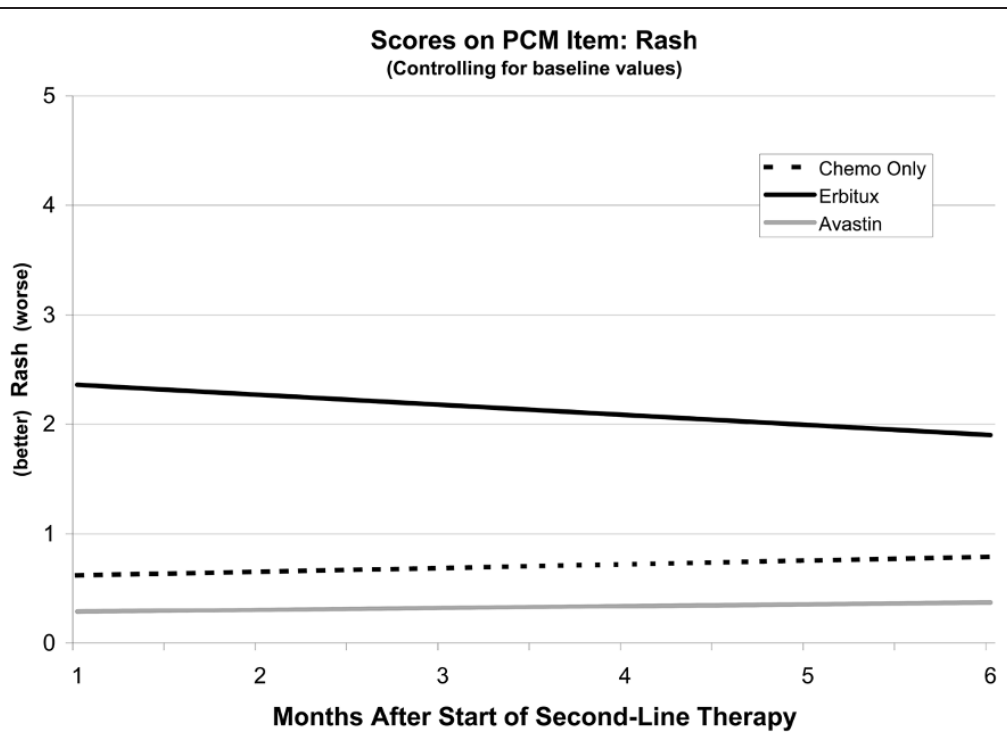

Figure $\mathbf{2}$ Linear mixed model of rash scores controlling for baseline.

contrast, Cetuximab patients had higher scores than patients receiving bevacizumab, regardless of whether or not we controlled for baseline values.

\section{Itching}

Itching scores tended to be stable across treatment groups (Figure 4). Baseline scores were a significant predictor in this model $(\mathrm{p}<.0001)$. Controlling for baseline scores, the Bevacizumab group scored significantly lower (better) than the Cetuximab group $(\mathrm{p}=.0002)$, and no different from the Chemotherapy Only group. As in the model for Dry Skin, baseline adjusted scores for the Chemotherapy Only group were comparable to those of the Bevacizumab group, but scores not adjusted for baseline were elevated compared to the Bevacizumab group. As with the analysis of Dry Skin, the implication is that the baseline scores of Chemotherapy Only patients, and not their group membership, dictated scores during second-line therapy. In contrast, for the Cetuximab patients, their membership in the Cetuximab group predicted higher scores during second-line chemotherapy, independent of their baseline scores.

\section{Nail changes}

There was a significant main effect of Interval $(\mathrm{p}=.0292)$, and a near significant interaction of Group with Interval

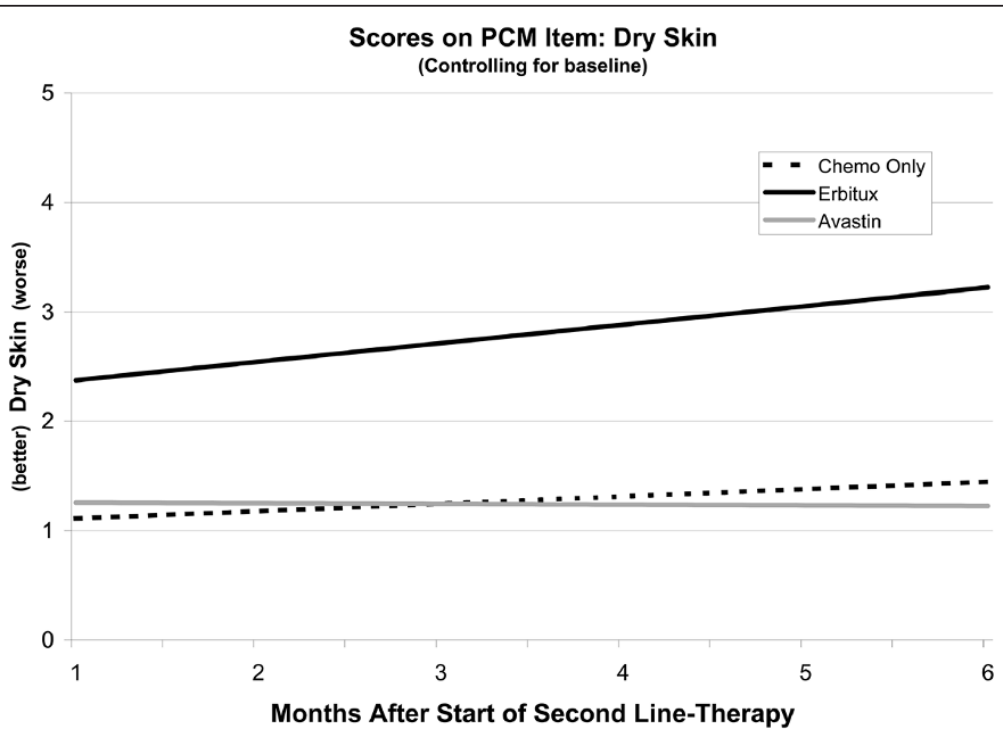

Figure 3 Linear mixed model of dry skin scores controlling for baseline. 


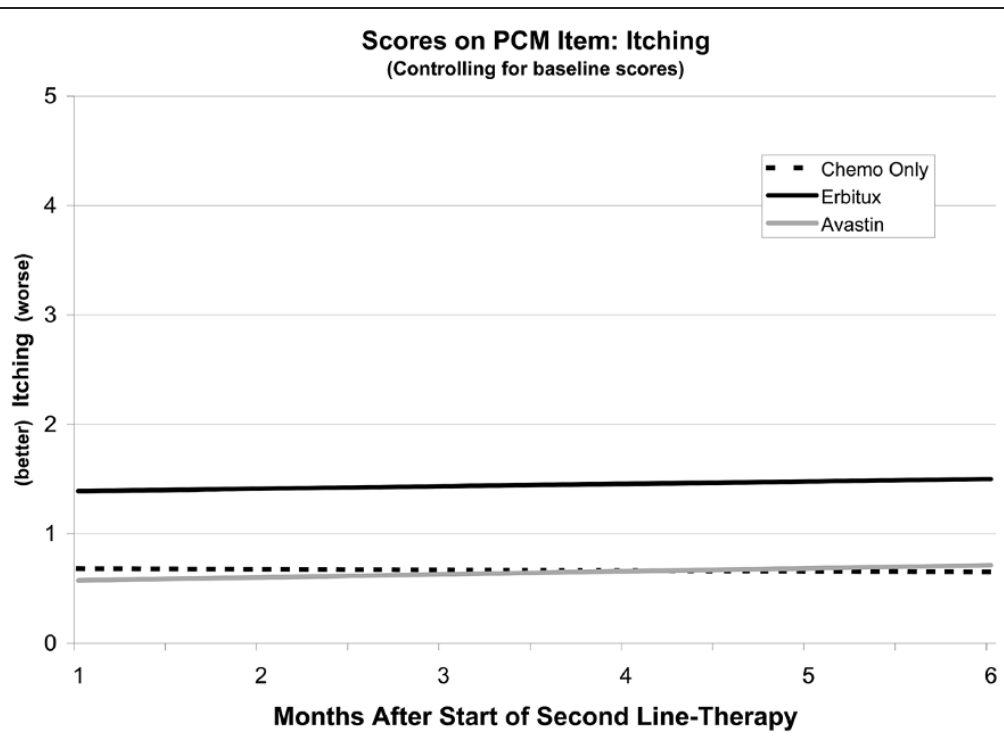

Figure 4 Linear mixed model of itching scores controlling for baseline.

( $\mathrm{p}=.0587)$ suggesting that the Bevacizumab and Chemotherapy Only groups basically had stable Nail Change scores that did not significantly differ, controlling for baseline. Conversely the Cetuximab group had scores that were increasing, representing a rate of change over time that was significantly greater than that of the Bevacizumab group $(\mathrm{p}=.0182)$. Baseline scores positively predicted scores during treatment $(\mathrm{p}<.0001)$.

\section{Nausea}

There was a significant but modest main effect of Interval $(\mathrm{p}=.0188)$, in which Nausea scores improved slightly over time. The effect represented less than 1 point improvement over a one year period with little notable difference among groups. Baseline scores did significantly predict Nausea scores over time during second-line treatment $(\mathrm{p}<.0001)$. There also was a small effect associated with duration of first-line treatment $(\mathrm{p}=.022)$, in which longer first-line treatment (e.g. 2 months) was associated with somewhat milder ( 0.1 point improvement) nausea symptoms during second-line therapy.

\section{Vomiting}

There was a significant but slight main effect of Interval $(\mathrm{p}=.0007)$, with Vomiting scores decreasing (improving) over time. However, the effect was very small (e.g. a 0.1 point improvement over a 5 month period, well short of a clinically significant effect), with very mild symptoms overall. There was no significance in the main effect of Group, or in the interaction of Group and Interval. Baseline scores strongly predicted Vomiting scores during treatment $(\mathrm{p}<.0001)$.

\section{Diarrhea}

There was a significant effect of Interval ( $\mathrm{p}<.0001)$, controlling for baseline, with scores improving over time. There was no significant main effect of Group, and no significant interaction between Interval and Group.

\section{Burning sensation in hands \& feet}

There were no significant main effects or interaction between Group and Interval on Scores for Burning Sensation in Hands \& Feet. Baseline scores did predict scores during second-line but with or without control of baseline, scores were relatively low, indicating mild symptoms, and relatively stable scores.

\section{PCM index scores}

Models assessing group differences on PCM index scores were also examined. In general, due to the composite nature of these measures, and the specificity of effects described in the foregoing section, any group differences were washed out on these endpoints. As noted earlier, the individual PCM items were the primary focus of the investigation.

\section{Discussion}

This study examined retrospectively collected medical record data, and repeated symptom burden assessments, from CRC patients with metastatic disease treated in the second-line setting with regimens containing Bevacizumab, Cetuximab, or Chemotherapy Only at seven community oncology practices in the United States (U.S.).

The results suggest that baseline symptom burden scores are the strongest single predictor of second-line 
symptom burden across the items measured in this study. However, after controlling for baseline symptom scores, the data suggest that patients on a Bevacizumab containing regimen tended to have more stable and less severe symptoms as measured by PCM items than patients on a Cetuximab containing regimen, and than patients on Chemotherapy Only in several areas.

This study adds to the literature in several ways. By assessing key symptoms with repeated assessments, this study was able to characterize symptom burden specifically during second-line therapy, after patients had received prior treatment and experienced disease progression. The study was able to directly compare symptom burden in key areas across regimens that included two widely used biologics, and across regimens that included chemotherapy only without monoclonal antibodies. The study was also able to show that some symptoms present at initiation of second-line therapy persist, and that symptoms assumed to reflect treatment group effects may be attributable to group differences in symptom burden at initiation of second-line therapy. The study was unable to identify obvious demographic or clinical differences between patients in the treatment groups. Given that there were group differences in self-reported symptom burden at start of second-line therapy, this suggests that these differences may not be easily identifiable other than by self-report, or that they arose during first-line therapy. Clinicians should be aware of both possibilities.

Elevated dermatological symptoms during second-line therapy were observed in both the Cetuximab and Chemotherapy Only groups, but were substantially explained by baseline scores for the Chemotherapy Only group. This was not true of the Cetuximab group, however. The data do not reveal why patients with elevated scores at baseline had these elevated scores-there were no identifiable differences across treatment groups in firstline regimen, or in the duration of first-line therapy. Regardless, the data do suggest that patients in the Chemotherapy Only group who did not have elevated rash and dry skin symptoms at baseline tended not to report such problems during second-line therapy, and that this claim is not supported for the Cetuximab group.

Although PCM index scores may be less sensitive to specific effects due to their composite nature, previous research has shown them to be sensitive endpoints in other cancer populations [26,30,31]. As noted, these were not the focus of the analysis, but given findings in prior research, the absence of findings for PCM index scores was somewhat unexpected.

In interpreting these findings, the following factors should be considered. First, although data were collected from a number of geographically dispersed oncology clinics in the U.S., the study used a convenience sample that may differ in unknown ways from the underlying population.
Also, the observational nature of this research involved comparison of existing groups, with the possibility that there were pre-existing group differences related to the study outcomes. For example, it is possible that patients treated with Bevacizumab may have had some unknown selection factor operating such that patients with fewer preexisting risk factors for skin related toxicities were also more likely to receive Bevacizumab than either Cetuximab or Chemotherapy Only. In this and other ways, selection bias may therefore have affected the direction and magnitude of group differences observed. A further limitation is that the comparison groups were unequal in sample size with the Bevacizumab group having 106 cases compared to the Cetuximab and Chemotherapy only groups with 38 cases each. This reduced the statistical power of comparisons among groups relative to a balanced study design. Finally, we examined patients with mCRC treated in community practice settings. Results may therefore not generalize to patients with other diseases, with disease at other stages, and to patients treated in other settings.

\section{Conclusions}

The biggest predictor of second-line symptom burden is the symptom burden observed at the end of the first-line regimen. Patients receiving a second-line therapy for $\mathrm{mCRC}$ which contained bevacizumab reported fewer treatment-related symptoms as compared to patients receiving a regimen containing cetuximab.

\section{Clinical practice points}

Treatment side effects common to systemic cancer therapy may be present at the start of second-line treatment of mCRC. The severity of these symptoms is predictive of the severity of symptoms patients will self-report as present during the course of second-line therapy. Although there are differences in the pattern of symptoms patients may experience across regimens and with different biologic therapies, differences in side effect profile across regimens during second-line $\mathrm{mCRC}$ may reflect baseline differences in symptom burden. These symptoms may be attributable to the first-line regimens received, and may not be evident other than through patient self-report. Additional discussion of symptom severity with patients may be useful in this regard.

\section{Competing interest}

The study reported in this paper was funded by Genentech, Inc. Elaine Yu, Pharm.D. and Yeun Mi Yim, M.P.H. are employed by and own stock in Genentech. Lee S. Schwartzberg, M.D. is a consultant for Roche. Genentech is a member of the Roche Group. The other authors report no conflicts of interest.

\section{Authors' contributions}

MW provided overall research design and scientific supervision. EY assisted with research design and scientific background. JK provided statistical analysis and quality control. YY provided background research and assisted with drafting the manuscript. ES provided research design consultation and 
measurement review. LS provided clinical background and assisted with research design and measurement decisions. All authors read and approved the final manuscript.

\section{Acknowledgements}

Funding for this study was provided by Genentech, Inc., South San Francisco, CA, USA. Special thanks to the staff of clinical research nurses at ACORN Research who assembled data for this study, and to Kathy L. Schulman, M.S. Outcomes Research Solutions, Inc., for preparation of the initial draft of the manuscript.

\section{Author details}

${ }^{1}$ ACORN Research, LLC, Memphis, TN, USA. ${ }^{2}$ Genentech, South San Francisco, CA, USA. ${ }^{3}$ The West Clinic, Memphis, TN, USA. ${ }^{4} 6555$ Quince, Suite 400, Memphis, TN 38119, USA.

Received: 29 May 2012 Accepted: 6 June 2012

Published: 20 June 2012

\section{References}

1. American Cancer Society: Colorectal Cancer Facts \& Figures 2011-2013 Atlanta. GA: American Cancer Society; 2011.

2. Coutinho AK, Rocha Lima CM: Metastatic colorectal cancer: systemic treatment in the new millennium. Cancer Control 2003, 10(3):224-238.

3. Rougier $\mathrm{P}$, Mitry E: Epidemiology, treatment and chemoprevention in colorectal cancer. Ann Oncol 2003, 14(Suppl 2):ii3-ii5.

4. Starling N, Tilden D, White J, Cunningham D: Cost-effectiveness analysis of cetuximab/irinotecan vs active/best supportive care for the treatment of metastatic colorectal cancer patients who have failed previous chemotherapy treatment. Br J Cancer 2007, 96(2):206-212.

5. Capdevila J, Ramos FJ, Macarulla T, Elez E, Tabernero J: The role of salvage treatment in advanced colorectal cancer. Crit Rev Oncol Hematol 2009, 71(1):53-61

6. Kelly H, Goldberg RM: Systemic therapy for metastatic colorectal cancer: current options, current evidence. J Clin Oncol 2005, 23(20):4553-4560.

7. The National Comprehensive Cancer Network: Clinical practice guidelines in oncology: colon cancer, version 3.2011. In The National Comprehensive Cancer Network: 2011

8. The National Comprehensive Cancer Network: Clinical practice guidelines in oncology: rectal cancer, version 4.2011. In The National Comprehensive Cancer Network: 2011.

9. ImClone and Bristol-Myers Squibb: Erbitux drug label. In ImClone and Bristol-Myers Squibb:; 2010.

10. Genentech: Drug label for Avastin. In Genentech.; 2009

11. Efficace F, Bottomley A, Coens C, Van Steen K, Conroy T, Schoffski $P$, et al: Does a patient's self-reported health-related quality of life predict survival beyond key biomedical data in advanced colorectal cancer? Eur J Cancer 2006, 42(1):42-49.

12. Efficace $F$, Innominato PF, Bjarnason $G$, Coens $C$, Humblet $Y$, Tumolo $S$, et al: Validation of patient's self-reported social functioning as an independent prognostic factor for survival in metastatic colorectal cancer patients: results of an international study by the Chronotherapy Group of the European Organisation for Research and Treatment of Cancer. J Clin Oncol 2008, 26(12):2020-2026.

13. Maisey NR, Norman A, Watson M, Allen MJ, Hill ME, Cunningham D: Baseline quality of life predicts survival in patients with advanced colorectal cancer. Eur J Cancer 2002, 38(10):1351-1357.

14. Feyer $P$, Kleeberg UR, Steingraber M, Gunther W, Behrens M: Frequency of side effects in outpatient cancer care and their influence on patient satisfaction-a prospective survey using the PASOOC questionnaire. Support Care Cancer 2008, 16(6):567-575.

15. Gupta D, Lis CG, Grutsch JF: The relationship between cancer-related fatigue and patient satisfaction with quality of life in cancer. J Pain Symptom Manage 2007, 34(1):40-47

16. Lis CG Gupta D, Grutsch JF. The relationship between insomnia and patient satisfaction with quality of life in cancer. Support Care Cancer 2008, 16(3):261-266

17. Osoba D, Hsu MA, Copley-Merriman C, Coombs J, Johnson FR, Hauber B, et al: Stated preferences of patients with cancer for health-related quality-of-life (HRQOL) domains during treatment. Qual Life Res 2006, 15(2):273-283.
18. Mittmann N, Seung SJ: Rash rates with EGFR inhibitors: meta-analysis. Curr Oncol 2011, 18(2):e54-e63.

19. Ouwerkerk J, Boers-Doets C: Best practices in the management of toxicities related to anti-EGFR agents for metastatic colorectal cancer. Eur J Oncol Nurs 2010, 14(4):337-349.

20. Fortner BV, Schwartzberg LS, Stepanski EJ, Houts AC: Symptom burden for patients with metastatic colorectal cancer treated with first-line FOLFOX or FOLFIRI with and without bevacizumab in the community setting. Supportive Cancer Therapy 2007, 4(4):233-240.

21. Conroy T, Hebbar M, Bennouna J, Ducreux M, Ychou M, Lledo G, et al: Quality-of-life findings from a randomised phase-III study of XELOX vs FOLFOX-6 in metastatic colorectal cancer. Br J Cancer 2010, 102(1):59-67.

22. Funaioli $C$, Longobardi C, Martoni AA: The impact of chemotherapy on overall survival and quality of life of patients with metastatic colorectal cancer: a review of phase III trials. J Chemother 2008, 20(1):14-27.

23. Fortner B, Okon T, Schwartzberg L, Tauer K, Houts AC: The Cancer Care Monitor: psychometric content evaluation and pilot testing of a computer administered system for symptom screening and quality of life in adult cancer patients. J Pain Symptom Manage 2003, 26(6):1077-1092.

24. Fortner B, Baldwin S, Schwartzberg L, Houts AC: Validation of the Cancer Care Monitor items for physical symptoms and treatment side effects using expert oncology nurse evaluation. J Pain Symptom Manage 2006, 31(3):207-214

25. Houts AC, Lipinski D, Olsen JP, Baldwin S, Hasan M: Use of the Patient Care Monitor to screen for depression in adult cancer patients interviewed with the structured clinical interview for DSM-IV. Psychooncology 2010, 19(4):399-407

26. Walker MS, Schwartzberg LS, Stepanski EJ, Fortner BV: A retrospective study of quality of life in a community sample of patients with early stage breast cancer. Breast Cancer Res Treat 2009, 115(2):415-422.

27. Stepanski EJ, Walker MS, Schwartzberg LS, Blakely LJ, Ong J, Houts AC: The relation of trouble sleeping, depressed mood, pain and fatigue in patients with cancer. J Clin Sleep Med 2009, 5(2):132-136.

28. Littell RC, Pendergast J, Natarajan R: Modelling covariance structure in the analysis of repeated measures data. Stat Med 2000, 19(13):1793-1819.

29. Cnaan A, Laird NM, Slasor P: Using the general linear mixed model to analyse unbalanced repeated measures and longitudinal data. Stat Med 1997, 16(20):2349-2380.

30. Houts A, Lalla D, Walker M, Stepanski E, Brammer M, Doan J, et al: Symptom burden declines when breast cancer patients treated with adjuvant trastuzumab/combination chemotherapy regimens enter trastuzumab monotherapy follow-up. In San Antonio Breast Cancer Symposium; 2010 December 8-12; San Antonio, TX; 2010

31. Walker MS, Stepanski EJ, Reyes C, Satram-Hoang S, Houts AC, Schwartzberg LS: Symptom burden and quality of life in patients with follicular lymphoma undergoing maintenance treatment with rituximab compared with observation. Ther Adv Hematol 2011, 2(3):129-139.

\section{doi:10.1186/1756-0500-5-314}

Cite this article as: Walker et al:: Symptom burden \& quality of life among patients receiving second-line treatment of metastatic colorectal cancer. BMC Research Notes 2012 5:314.

\section{Submit your next manuscript to BioMed Central and take full advantage of:}

- Convenient online submission

- Thorough peer review

- No space constraints or color figure charges

- Immediate publication on acceptance

- Inclusion in PubMed, CAS, Scopus and Google Scholar

- Research which is freely available for redistribution 